\title{
RATAN-600 Observations of Unusual Inversion of Polarization in Sunspot Associated Microwave Sources
}

\author{
N. A. B. Gizani \\ Institute of Astronomy $\&$ Astrophysics, National Observatory of Athens, \\ I. Metaxa 85 B. Paulou, Lofos Koufou, Penteli, Athens \\ C. Alissandrakis \\ University of Ioannina, Section of Astrogeophysics, GR-45110 Ioannina, \\ Greece \\ V. Bogod \& V. Garaimov \\ Special Astrophysical Observatory, 357147, Karachaevo-Cherkessia, \\ Russia \\ V. Zheleznyakov \\ Pulkovo Astronomical Observatory, 196140, Pulkovo, Russia \\ E. Zlotnik \\ Institute of Applied Physics, 603600 Nizhnij Novgorod, Russia
}

\section{Summary}

We have discovered unusual inversion of circular polarization in both components of an active region. We have observed the region for six consecutive days in May 96 using the RATAN-600 radio telescope with high polarization accuracy up to $0.5 \%$. The observations were taken in the range of 1.7 to $30 \mathrm{~cm}$. At short wavelengths the circular polarization scans revealed a bipolar structure, expected since the magnetic field is bipolar. However, for all six days and while the region was moving from the center of the disk to the west limb, the circular polarization dropped practically to zero at $14.5 \mathrm{~cm}$ and at longer wavelengths the sense of the polarization was reversed.

We show that this unusual polarization inversion cannot be interpreted in terms of the known propagation effects under conditions of weak and strong coupling. Linear coupling could explain the observed polarization only if the magnetic field was parallel to a solar meridian (i.e. perpendicular to the magnetic dipole axis of the active region). Then the polarization would be independent of the solar rotation and the reversal would occur for both group components at the same frequency. This field could be the very large scale, global solar magnetic field, but its existence is not confirmed observationally (EUV and soft X-ray). The most likely interpretation is the change of the predominant emission mechanism near the wavelength of polarization reversal. The change from thermal at shorter wavelengths to non-thermal at longer ones could cause the predominance of o-mode emission at lower frequencies. 